\title{
Prognosis in patients with transient ischaemic attack (TIA) and minor stroke attending TIA services in the North West of England: The NORTHSTAR Study
}

\author{
J R Selvarajah,, ${ }^{1}$ C J Smith, ${ }^{2}$ S Hulme, ${ }^{2}$ R F Georgiou, ${ }^{2}$ A Vail, ${ }^{3}$ P J Tyrrell, ${ }^{2}$ on behalf of \\ the NORTHSTAR Collaborators
}

${ }^{1}$ Department of Neurology, Leeds General Infirmary, Leeds, UK; ${ }^{2}$ Clinical Neurosciences Group, University of

Manchester, Clinical Sciences Building, Hope Hospital, Salford, UK; ${ }^{3}$ Biostatistics Group, University of Manchester, Clinical Sciences Building, Hope Hospital, Salford, UK

Correspondence to:

Dr C J Smith, Clinical

Neurosciences Group, University of Manchester, Clinical Sciences Building, Hope Hospital, Salford M6 8HD, UK; Craig.Smith-2@ manchester.ac.uk

Received 12 July 2007 Revised 29 August 2007 Accepted 6 September 2007

\section{ABSTRACT}

Background: The $A B C D^{2}$ score predicts stroke risk within a few days of transient ischaemic attack (TIA). It is not clear whether the predictive value of the $A B C D^{2}$ score can be generalised to UK TIA services, where delayed presentation of TIA and minor stroke are common. We investigated prognosis, and the use of the $A B C D^{2}$ score, in patients attending TIA services in the North West of England with a diagnosis of TIA or minor stroke.

Methods: 711 patients with TIA or minor stroke were prospectively recruited from five centres (median duration from index event to recruitment 15 days). The primary outcome was the composite of incident TIA, stroke, acute coronary syndrome or cardiovascular death at the 3 month follow-up. Prognostic factors were analysed using Cox proportional hazards regression.

Results: The primary outcome occurred in $126(18 \%)$ patients. Overall, there were 30 incident strokes. At least one incident TIA occurred in 100 patients (14\%), but only four had a subsequent stroke. In multifactorial analyses, the $A B C D^{2}$ score was unrelated to the risk of the primary outcome, but predicted the risk of incident stroke: score 4-5: hazard ratio (HR) 3.4 (95\% Cl 1.0 to 12); score 6-7: HR 4.8 (1.3 to 18). Of the components of the $A B C D^{2}$ score, unilateral motor weakness predicted both the primary outcome (HR 1.8 (1.2 to 2.8)) and stroke risk (HR 4.2 (1.3 to 14)).

Conclusions: In patients attending typical NHS TIA services, the risk of incident stroke was relatively low, probably reflecting delays to assessment. Current provision of TIA services, where delayed presentation to "rapid access" TIA clinics is common, does not appear to provide an appropriate setting for urgent evaluation, risk stratification or timely secondary prevention for those who may be at highest risk.

Several studies have highlighted that the early risk of stroke after transient ischaemic attack (TIA) is higher than previously thought. In population or hospital based studies of patients within a few days of TIA, the risk of stroke is approximately $5 \%$ at 2 days, $7-10 \%$ at 7 days and $10-17 \%$ at 3 months. ${ }^{1-4}$ Furthermore, patients with TIA are also at risk of subsequent cardiovascular events. ${ }^{5}$ When considering composite vascular end points after TIA, the risks of stroke, recurrent TIA, cardiac hospitalisation or death are as high as $25 \%$ at 3 months. ${ }^{1}$

Recent studies have sought to identify predictors of early stroke risk in order to inform risk stratification and clinical decision making in the assessment of patients with TIA. The California score predicted 90 day stroke risk in patients attending the emergency room with probable TIA, of which nearly all were within $24 \mathrm{~h}$ of symptom onset. ${ }^{1}$ The $\mathrm{ABCD}$ score predicted 7 day stroke risk in both a population based TIA referral service and a hospital based TIA clinic where the majority of patients were seen within a week of TIA. ${ }^{6}$ More recently, the $\mathrm{ABCD}^{2}$ score, derived from the California and $A B C D$ scores, has been validated in these cohorts, predicting the risk of stroke at 2,7 or 90 days. $^{7}$ The $\mathrm{ABCD}^{2}$ score is based on five components: age ( $\geqslant 60$ years $=1$ point), blood pressure (systolic $\geqslant 140 \mathrm{~mm} \mathrm{Hg}$ and/or diastolic $\geqslant 90 \mathrm{~mm} \mathrm{Hg}=1$ point), clinical features (unilateral weakness $=2$ points; speech disturbance without weakness $=1$ point), duration of symptoms in minutes $(\geqslant 60=2$ points; 10 $59=1$ point) and diabetes (present $=1$ point). The $\mathrm{ABCD}^{2}$ score has a minimum score of 0 and a maximum score of 7 .

The approach to management of TIA and minor stroke patients, either by referral to "rapid access" TIA outpatient clinics or admission to hospital for further investigation and management, remains variable and controversial. ${ }^{8}{ }^{9}$ Current UK guidelines advocate specialist assessment and investigation of all TIA patients within 7 days of symptom onset. ${ }^{10}$ However, this is currently only achieved in 35\% of UK centres and the nationwide median delay from referral to specialist TIA clinic appointment is 12 days. ${ }^{11}$ The $\mathrm{ABCD}^{2}$ and related scores were developed in populations where the majority presented within $24 \mathrm{~h}$ of TIA symptom onset. It is not clear whether the predictive value of the $\mathrm{ABCD}^{2}$ score can be generalised to UK TIA services, where delayed presentation of both TIA and minor stroke are common. The aim of the present study was to investigate the 3 month prognosis of patients with confirmed TIA or minor stroke assessed in a representative sample of NHS TIA services, and evaluate the use of the $\mathrm{ABCD}^{2}$ score in predicting incident vascular events, specifically from the time of attendance. To achieve this aim, we performed secondary analyses of data collected prospectively in the North West of England Transient Ischaemic Attack and Minor Stroke (NORTHSTAR) study cohort.

\section{METHODS}

The NORTHSTAR Study is a prospective, multicentre, nested, case control study designed to investigate the prognostic role of peripheral and genetic inflammatory markers in patients with 
Table 1 Baseline characteristics and medications at study entry $(\mathrm{n}=711)$

\begin{tabular}{|c|c|}
\hline \multicolumn{2}{|l|}{ Index diagnosis } \\
\hline Cerebral TIA & $311(43.7)$ \\
\hline Cerebral minor stroke & $301(42.3)$ \\
\hline Retinal TIA & $63(8.9)$ \\
\hline BRAO/CRAO & $36(5.1)$ \\
\hline \multicolumn{2}{|l|}{ Duration of symptoms (min) } \\
\hline$\geqslant 60$ & $439(61.7)$ \\
\hline $10-59$ & $146(20.5)$ \\
\hline$<10$ & $126(17.7)$ \\
\hline Age $(y)^{*}$ & $68(27,93)$ \\
\hline Male & $417(58.6)$ \\
\hline White Caucasian & $704(99.0)$ \\
\hline TIA or minor stroke in preceding month & $127(17.9)$ \\
\hline Any previous stroke or TIA & $284(39.9)$ \\
\hline Coronary artery disease & $182(25.6)$ \\
\hline Hypertension & $498(70.0)$ \\
\hline Diabetes mellitus & $118(16.6)$ \\
\hline Dyslipidaemia & $512(72.0)$ \\
\hline Peripheral vascular disease & $81(11.4)$ \\
\hline Atrial fibrillation & $72(10.1)$ \\
\hline Left ventricular hypertrophy & $77(11.2) n=689$ \\
\hline \multicolumn{2}{|l|}{ Smoking history } \\
\hline Current smoker & $230(32.3)$ \\
\hline Ex smoker & $293(41.2)$ \\
\hline Never smoker & $188(26.4)$ \\
\hline Alcohol intake (units per week)* & $4(0,126) n=682$ \\
\hline Body mass index $\left(\mathrm{kg} / \mathrm{m}^{2}\right)^{*}$ & $27.1(14.8,55.0) n=708$ \\
\hline $\mathrm{BP}(\mathrm{mm} \mathrm{Hg}) \dagger$ & $149 / 81(24 / 13)$ \\
\hline \multicolumn{2}{|l|}{ Carotid imaging } \\
\hline Occlusion of at least one ICA & $34(5.2)$ \\
\hline $70-99 \%$ stenosis of at least one ICA & $90(13.7)$ \\
\hline \multicolumn{2}{|l|}{ Medications at study entry } \\
\hline Aspirin & $523(73.6)$ \\
\hline Other antiplatelet agent & $151(21.2)$ \\
\hline Anticoagulation & $37(5.2)$ \\
\hline Statin & $387(54.4)$ \\
\hline ACE-I & $205(28.9)$ \\
\hline All RB & $73(10.3)$ \\
\hline Other antihypertensive & $355(49.9)$ \\
\hline Diabetes treatment & $78(11.0)$ \\
\hline
\end{tabular}

Values are $\mathrm{n}(\%)$ except ${ }^{*}$ median (minimum, maximum) and tmean (SD).

ACE-I, angiotensin converting enzyme inhibitor; AllRB, angiotensin II receptor blocker; $\mathrm{BP}$, blood pressure; $\mathrm{BRAO}$, branch retinal artery occlusion; $\mathrm{CRAO}$, central retinal artery occlusion; ICA, internal carotid artery; TIA, transient ischaemic attack.

recent TIA or minor stroke. The primary analyses of the NORTHSTAR Study will be reported separately. The study was conducted in TIA services at five UK centres (see appendix) in Greater Manchester and Liverpool. Local research ethics committee approval for the study was obtained for each centre. Four centres each operated a weekly TIA clinic, and one operated twice weekly clinics. Referrals to clinics were from primary care, accident and emergency, and other hospital specialties, including ophthalmology services. In addition, some patients were recruited directly from the accident and emergency unit or the acute stroke unit at Hope Hospital, Salford. The eligibility criteria for inclusion were diagnosis of TIA or minor stroke confirmed by a consultant stroke physician or neurologist, symptom onset within the preceding 6 weeks, age $\geqslant 18$ years, modified Rankin Scale (mRS) $\leqslant 1$, no known cognitive impairment sufficient to interfere with independent daily living, no significant comorbidity limiting participation in the study and intention to remain resident within the area for the duration of the study, with access to a telephone. TIA was defined as a clinical syndrome of acute loss of focal cerebral or monocular function, of vascular origin, with symptoms resolving within $24 \mathrm{~h} .{ }^{12}$ Minor stroke was defined as a clinical syndrome of rapidly developing symptoms and signs of focal loss of cerebral function, of vascular origin, lasting more than $24 \mathrm{~h},{ }^{13}$ with minimal residual functional impairment (mRS $\leqslant 1) \cdot{ }^{14}$

Patients with a confirmed diagnosis of TIA or minor stroke were screened for eligibility by a member of the research team. Written informed consent was obtained in all cases. Baseline data, including date and duration of symptoms, past medical history, vascular risk factors, current medications, $\mathrm{mRS},{ }^{15}$ Barthel Index (BI), ${ }^{16}$ blood pressure (BP) and ECG were recorded at study entry. A history of atrial fibrillation was defined by current atrial fibrillation on ECG using standard criteria or previously documented atrial fibrillation. Left ventricular hypertrophy was assigned using the Minnesota guidelines (codes 3-1 and/or 3-3). ${ }^{17}$ TIA and minor stroke were classified as either cerebral or retinal. Cerebral events were further classified as anterior or posterior circulation, based on the clinical features, supplemented by imaging findings where possible. Following recruitment, investigation and secondary prevention were initiated at the discretion of the clinician responsible for the patient. The study entry $\mathrm{ABCD}^{2}$ score was assigned retrospectively using clinical information collected at the time of recruitment. ${ }^{7}$

\section{Follow-up}

At recruitment, contact details of the research team were given to patients, relatives and their general practitioner, and attached to the medical notes. Patients were requested to report any changes in their health. Prior to the 3 month follow-up assessment, a survival check was undertaken using the tracking system of the NHS Central Register, hospital records or general practitioner records. Surviving patients were contacted by telephone, or less frequently interviewed in person, using a standardised questionnaire approximately 3 months after the index event. The questionnaire comprised the $\mathrm{mRS}, \mathrm{BI}$, medical and surgical history review and vascular event screening

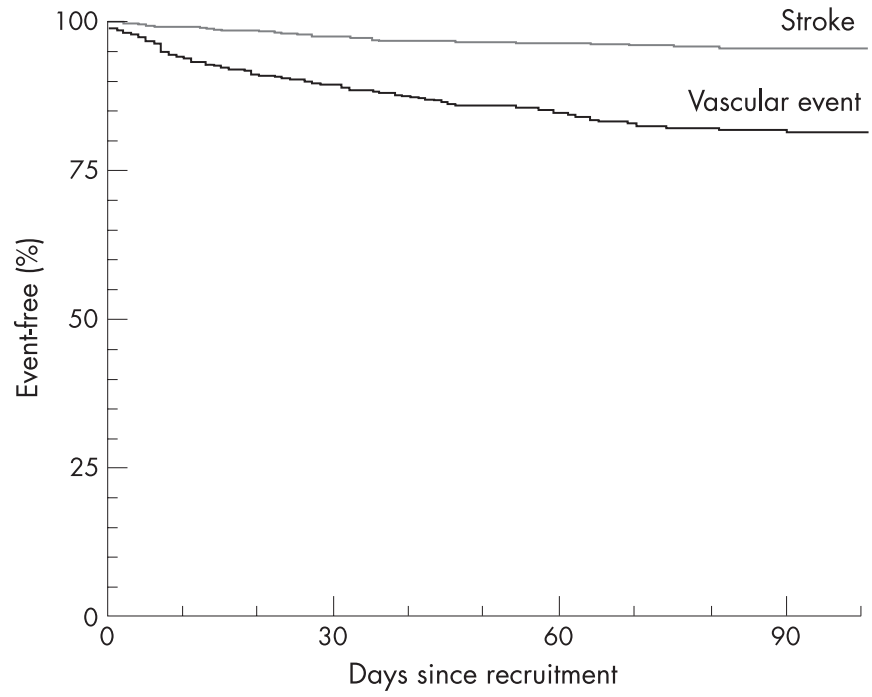

Figure 1 Kaplan-Meier curves illustrating survival free from acute vascular events and stroke from time of presentation to transient ischaemic attack services. 
Table 2 Primary outcome events, subsequent acute vascular events and natural history of primary outcome events by the 3 month follow-up $(\mathrm{n}=709)$

\begin{tabular}{lc}
\hline Primary outcome event $(n=126)$ & $100(79.4)$ \\
TIA & $25(19.8)$ \\
Non-fatal stroke & $1(0.8)$ \\
Cardiovascular death & \\
Natural history of primary outcome events $(n=126)$ & $96(76.2)$ \\
TIA only & 62 \\
1 TIA & 34 \\
$\geqslant 2$ TIA & $4(3.2)$ \\
TIA then stroke* & 2 \\
TIA then stroke & 1 \\
TIA then fatal stroke & 1 \\
2 TIAs then stroke & $25(19.8)$ \\
Stroke only & 24 \\
1 stroke & 1 \\
2 strokes & $1(0.8)$ \\
Cardiovascular death & \\
\hline
\end{tabular}

Values are $\mathrm{n}(\%)$.

${ }^{*}$ Fatal or non-fatal.

TIA, transient ischaemic attack.

questionnaire, including stroke-free status. ${ }^{18}$ For consistency, all follow-up interviews were conducted by the same research physician. Information obtained directly from patients and/or relatives was supplemented, wherever possible, by hospital records, general practitioner records and death certification. Follow-up was continued until death, or 3 months from the date of the index event.

\section{Definition of outcome events}

The primary outcome was time to the predefined composite end point of first verified incident TIA, fatal or non-fatal stroke, acute coronary syndrome or cardiovascular death during followup. Acute coronary syndromes were defined according to WHO guidelines. ${ }^{19}$ Cardiovascular death was defined by acute coronary syndrome certified as the cause of death or contributing directly to death. Fatal stroke was defined as incident stroke as the cause of death or contributing directly to death. All suspected outcome events were adjudicated by a panel (see appendix) of experienced consultant physicians (one stroke physician, one vascular neurologist and one elderly care physician) appointed prior to study commencement, and required agreement of all three panel members. In patients with more than one verified outcome event, the first incident event during follow-up was assigned the primary outcome. Incident strokes occurring during follow-up were analysed separately.

\section{Statistical analysis}

Time from recruitment until primary outcome event, stroke or end of follow-up was analysed using Cox proportional hazards regression in SPSS version 13.0. The assumption of proportionality was checked using the complementary log-log plot and found to be acceptable for the risk factors of interest. It was assumed that the hazard of further acute vascular events would be highest immediately following TIA or minor stroke and then decrease. Therefore, all analyses controlled for whether participants had experienced an acute cerebrovascular event in the month preceding the index event and for the time from the index event until recruitment. Analyses of risk factors for subsequent stroke were adjusted only for these two confounders because of the low number of strokes observed. Analyses of risk factors for the primary outcome were adjusted simultaneously for other putative risk factors.

\section{RESULTS}

A total of 711 patients meeting the eligibility criteria were recruited between June 2003 and January 2006. Baseline characteristics are presented in table 1. Median (range) duration from the index TIA or minor stroke to study entry was 15 (042) days. Seventy-eight patients (11\%) were inpatients when recruited; the remainder were recruited from TIA outpatient clinics. Clinical features of the index event were as follows: unilateral weakness (54\%), speech disturbance (42\%), hemisensory disturbance (38\%), monocular visual loss $(14 \%)$, binocular visual loss (11\%), ataxia (13\%) and vertigo $(3 \%)$. Subtypes were classified as follows: anterior circulation 611 $(86 \%)$, posterior circulation 100 (14\%). Brain imaging was undertaken in 481 (68\%) patients and carotid duplex, with or without magnetic resonance angiography, in 656 (92\%). Two patients were lost to follow-up immediately after recruitment. The other 709 patients had known status through to at least 6 weeks following recruitment. Forty-seven patients (7\%) underwent carotid endarterectomy during follow-up.

The primary outcome occurred in 126 patients (18\%) (fig 1, table 2), of whom 28 were hospitalised. A further 59 outcome events in 39 participants occurred (table 2). Overall, there were 30 fatal or non-fatal incident strokes in 29 (4\%) of the cohort (fig 1) and stroke accounted for nearly $20 \%$ of the primary outcome events. In contrast, at least one incident TIA occurred in $100(14 \%)$ of the cohort, but only four of these patients went on to have a stroke during follow-up. There were three deaths during follow-up, two of which were vascular deaths (a perioperative fatal stroke at carotid endarterectomy and an acute coronary syndrome). Of 706 survivors followed-up to 3 months, 508 (72\%) were mRS grade 0 and 188 (27\%) mRS grade 1. Six hundred and eighty-eight (97\%) patients scored 95 or 100 on the BI.

In multifactorial analyses, the $\mathrm{ABCD}^{2}$ score was unrelated to the risk of the primary outcome, despite significant association with the risk of incident stroke (fig 2, table 3). Hazard ratios (95\% CI) for the individual components of the $\mathrm{ABCD}^{2}$ score for stroke were: age $\geqslant 60$ years, 0.93 (0.41 to 2.1$)$; $\mathrm{BP} \geqslant 140 / 90,0.89$ (0.43 to 1.9$)$; speech disturbance without weakness, 1.9 (0.38 to 9.3); unilateral weakness, 4.2 (1.3 to 14$)$; symptom duration at least $1 \mathrm{~h}, 4.5$ (1.5 to 14 ); and diabetes, 0.80 (0.28 to 2.3). Thus the relationship between $\mathrm{ABCD}^{2}$ score and stroke risk appeared to be driven by the presence of unilateral weakness and symptom duration of $1 \mathrm{~h}$ or more. Of all components, only unilateral weakness was found to significantly increase the risk of the subsequent primary outcome event (HR 1.8 (95\% CI 1.2 to 2.8$)$ ).

\section{DISCUSSION}

We evaluated prognosis in a large prospective cohort of patients attending TIA services in the North West of England with confirmed TIA or minor stroke, where the majority of patients were assessed in "rapid access" TIA outpatient clinics. The median delay from onset of the index event to assessment was 15 days, which is representative of current UK practice. ${ }^{11}$ Our eligibility criteria were pragmatic, but excluded patients with pre-existing significant physical disabilities and/or cognitive impairment. Despite this, we believe our findings can be generalised to TIA services across the UK, because all are likely 

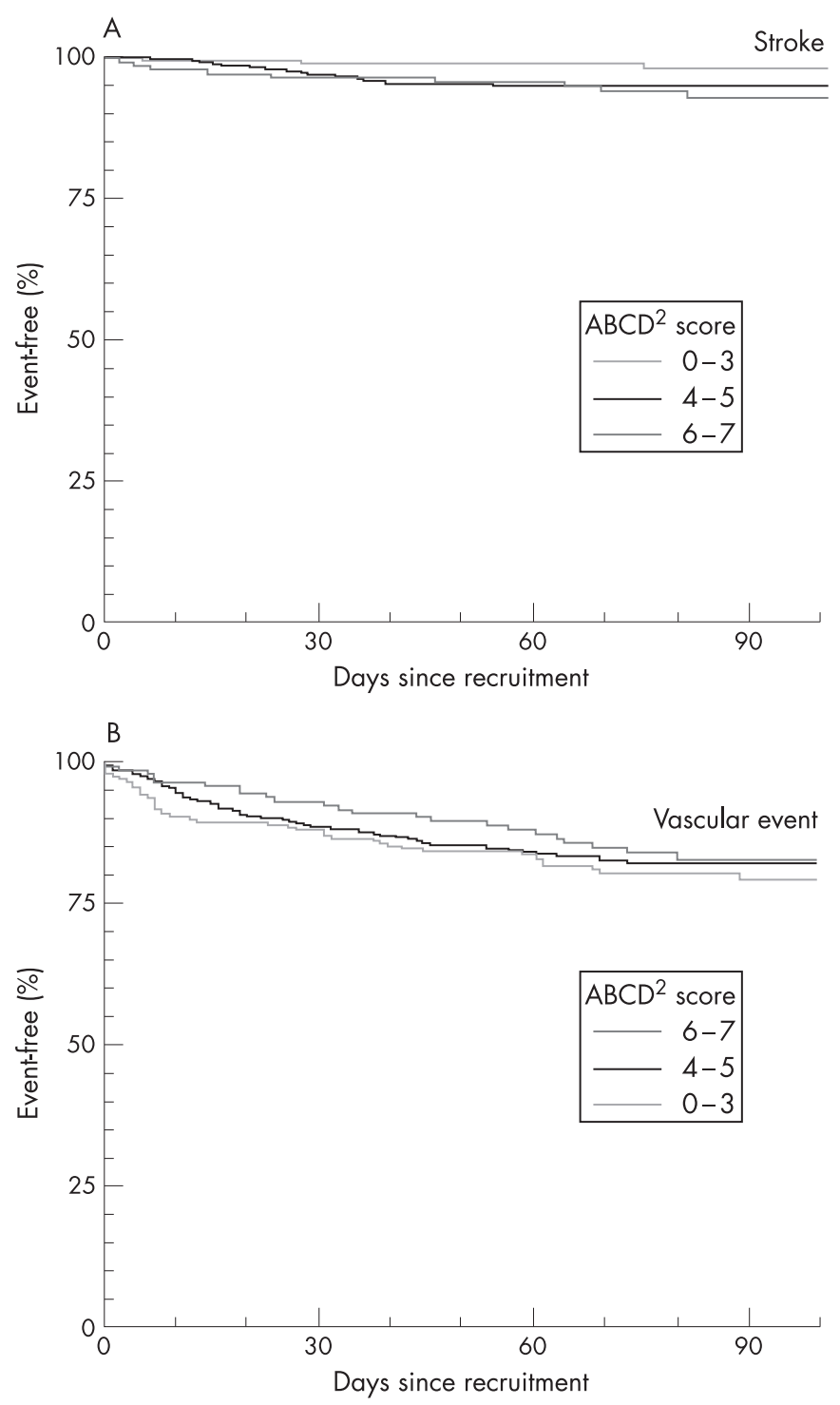

Figure 2 Kaplan-Meier curves illustrating survival free from (A) stroke and $(B)$ acute vascular events from time of presentation to transient ischaemic attack services, stratified according to $A B C D^{2}$ score.

to include many similar patients to ours, recruited with similar delays.

Our estimate of 3 month stroke risk is less than half that reported in most studies where patients were assessed within the first few days of TIA onset. ${ }^{1-3}$ In these studies, around half of the strokes at 3 months had occurred within 2 days of the index TIA, at a time before most of the present cohort were recruited. Despite this, the 3 month stroke risk in the present study was still comparable with some studies assessing patients soon after the index TIA. ${ }^{20}{ }^{21}$ Furthermore, the risk of composite vascular end points in our study was still relatively high at 3 months, which accords with previous studies. ${ }^{14}$ A high proportion of our patients had a TIA or minor stroke in the month preceding the index event. However, we did not ascertain the proportion of patients initially referred to TIA services who then had a stroke, and subsequently did not attend. It is noteworthy that the majority of our patients were already taking at least one antiplatelet agent, or anticoagulants, and approximately $54 \%$ were taking statins at study entry, which may have influenced incident stroke rates. We did not collect data on the duration of these therapies prior to study entry, but following study entry, additional antiplatelet agents were prescribed in a further $37 \%$ and statins in a further $35 \%$ (data not shown).

The $\mathrm{ABCD}^{2}$ score predicted the 3 month stroke risk in our cohort, despite the relatively low rate of stroke and delay from index event to assessment. Our findings suggest that it may be reasonable to apply the $\mathrm{ABCD}^{2}$ score in patients with minor stroke, not only in those with TIA, although we did not analyse the two groups separately. From a practical point of view, this is relevant as the risk of incident stroke is comparable in patients with TIA and minor stroke, ${ }^{2}$ and patients with minor stroke accounted for around half of our cohort. When analysing the components of the $\mathrm{ABCD}^{2}$ score separately, only unilateral motor weakness and symptom duration predicted stroke. This accords with recent evidence suggesting that these clinical features, along with dysphasia or dysarthria, are more likely to be associated with underlying cerebral ischaemic lesions on diffusion weighted imaging. ${ }^{22}$ We used clinical information collected at the time of presentation to TIA services to assign the $\mathrm{ABCD}^{2}$ score, which is useful if patients did not initially seek medical attention soon after their event. However, this may explain why BP, recorded following delayed presentation, was not associated with stroke risk in our study. It is not clear why age, speech disturbance or diabetes mellitus were not associated with stroke risk, but there was a lack of statistical power given the low number of incident strokes. It is possible that at risk older patients had their stroke prior to attending TIA services. However, several other studies have also not identified associations between diabetes, ${ }^{321}$ age $^{3421}$ or certain clinical features and risk of stroke 3 months after TIA. ${ }^{21}$

As the $\mathrm{ABCD}^{2}$ score was designed to predict early stroke risk after TIA, it is therefore perhaps not surprising that it was unrelated to the risk of the composite primary outcome in the present study. However, unilateral weakness did predict

Table 3 Risk factors for the primary outcome or stroke from Cox regression models

\begin{tabular}{lllll}
\hline Risk factor & Level & $\mathbf{n}$ & Primary outcome event & Stroke ${ }^{\dagger}$ \\
\hline Time since index event & Per day & 711 & $0.99(0.97$ to 1.0$)$ & $0.97(0.94$ to 1.0$)$ \\
TIA or minor stroke in preceding month & Yes & 127 & $2.7(1.9$ to 4.0$)$ & $0.82(0.31$ to 2.2$)$ \\
ABCD $^{2}$ score & 0 to 3 & 210 & 1.0 & 1.0 \\
& 4 or 5 & 356 & $0.89(0.60$ to 1.3$)$ & $3.4(1.0$ to 12$)$ \\
& 6 or 7 & 145 & $0.93(0.55$ to 1.6$)$ & $4.8(1.3$ to 18$)$ \\
Smoking & Ex & 293 & $1.5(0.91$ to 2.6$)$ & $1.8(0.50$ to 6.8$)$ \\
& Current & 230 & $2.4(1.5$ to 4.1$)$ & $4.3(1.3$ to 15$)$
\end{tabular}

${ }^{*}$ Single multifactorial model including all factors (HR $(95 \% \mathrm{CI})$ ).

$\dagger$ Separate multifactorial models each containing the first two factors (HR $(95 \% \mathrm{CI})$ ).

TIA, transient ischaemic attack. 
incident stroke or the composite primary outcome, but we did not examine factors predicting risk of incident TIA as a separate outcome. In contrast with the risk of stroke at 3 months, the risk of one or more incident TIA following the index event was considerably higher, but comparable with previous studies. ${ }^{23}$ Only a minority of these patients went on to have a subsequent stroke, suggesting that a subset of patients with recurrent TIA presenting to TIA services have a relatively benign short term outcome. Further studies are required to refine risk stratification of patients presenting with recurrent TIAs, to differentiate those at risk of stroke from those with a more benign prognosis.

Our findings have relevance when considering current UK practice. Although patients attending typical TIA services are generally at low risk of incident stroke in the short term, the $\mathrm{ABCD}^{2}$ score can be used to identify patients at risk of stroke, and might be applied to inform targeted expedited investigation and secondary prevention in these individuals. However, our data suggest that current provision of TIA services where delayed presentation to "rapid access" TIA clinics is common, does not appear to provide an appropriate setting for urgent evaluation or timely secondary prevention in those who may be at the highest risk of stroke. Our findings therefore support the argument that patients with TIA or minor stroke require more urgent evaluation and risk stratification, requiring major improvements in public awareness and prompt primary care/ accident and emergency triage. ${ }^{24}{ }^{25}$ In high risk patients triaged acutely using the $\mathrm{ABCD}^{2}$ score, more effective stroke prevention might be achieved by admission to hospital or by provision of daily open access TIA clinics, but these strategies require further evaluation and may prove challenging to implement. Traditional "rapid access" TIA clinics would still continue to play a valuable role in the differential diagnosis and management of TIA presentations, perhaps with more focus on management of lower risk patients triaged acutely using the $\mathrm{ABCD}^{2}$ score, who would still require specialist assessment within 7 days, ${ }^{10}$ and patients with delayed presentations to medical attention.

The present study has several limitations. The analyses presented here were secondary analyses of a prospective cohort, with the $\mathrm{ABCD}^{2}$ score assigned retrospectively. As patient assessments were generally delayed following the index event, recall of symptoms and durations may have been less reliable in some cases. Not all patients underwent brain imaging, which was at the discretion of individual treating stroke physicians and neurologists. This variation reflects current UK practice and the lack of specific guidelines regarding brain imaging following TIA or minor stroke. Finally, although we did not perform faceto-face follow-up interviews in all instances, telephone followup was attempted in all patients. However, we did use a single interviewer, a validated questionnaire and independently verified vascular events.

\section{CONCLUSION}

In patients with TIA or minor stroke presenting to typical UK TIA services, the risk of incident stroke at 3 months was relatively low, probably reflecting delays in patient assessment. The $\mathrm{ABCD}^{2}$ score predicted the incident 3 month stroke risk, but not composite vascular end points. Incident TIA occurred more frequently, and generally followed a benign course. Current provision of TIA services, where delayed presentation to "rapid access" TIA clinics is common, does not appear to provide an appropriate setting for urgent evaluation, risk stratification or timely secondary prevention in those who may be at the highest risk of stroke.
Acknowledgements: We are extremely grateful to Professor Nancy J Rothwell and Dr Stephen J Hopkins for their intellectual input during the design and analyses of the NORTHSTAR study, and our colleagues and the staff in the collaborating TIA services for their assistance throughout the study (see appendix).

PJ Tyrrell is the Principal Investigator of The NORTHSTAR study and designed the study with CJS, AV and RFG. AV was responsible for the statistical analyses. JRS, SH and CJS collected the data. JRS, CJS and AV analysed the data. All authors contributed to writing the manuscript.

Funding: The study was funded by The Sir Jules Thorn Charitable Trust. J R Selvarajah was funded by The Sir Jules Thorn Charitable Trust. C J Smith is funded by The Stroke Association. S Hulme and R F Georgiou are funded by The Sir Jules Thorn Charitable Trust. A Vail is funded by The Salford Royal NHS Foundation Trust and P J Tyrrell is funded by the University of Manchester. The funding source had no role in study design, data collection, analyses or interpretation of the data, writing the manuscript or in the decision to submit the manuscript for publication.

Competing interests: None.

\section{REFERENCES}

1. Johnston SC, Gress DR, Browner WS, et al. Short-term prognosis after emergency department diagnosis of TIA. JAMA 2000;284:2901-6.

2. Coull AJ, Lovett JK, Rothwell PM. Population based study of early risk of stroke after transient ischaemic attack or minor stroke: implications for public education and organisation of services. BMJ 2004;328:326-8.

3. Hill MD, Yiannakoulias N, Jeerakathil T, et al. The high risk of stroke immediately after transient ischaemic attack: a population-based study. Neurology 2004;62:201520.

4. Kleindorfer D, Panagos P, Pancioli A, et al. Incidence and short-term prognosis of transient ischaemic attack in a population-based study. Stroke 2005;36:720-3.

5. Touzé $\mathbf{E}$, Varenne 0 , Chatellier $\mathrm{G}$, et al. Risks of myocardial infarction and vascular death after transient ischaemic attack and ischaemic stroke: a systematic review and meta-analysis. Stroke 2005;36:2748-55.

6. Rothwell PM, Giles MF, Flossmann E, et al. A simple score (ABCD) to identify individuals at high early risk of stroke after transient ischaemic attack. Lancet 2005;366:29-36.

7. Johnston SC, Rothwell PM, Nguyen-Huynh MN, et al. Validation and refinement of scores to predict very early stroke risk after transient ischaemic attack. Lancet 2007;369:283-92.

8. Hill MD, Gladstone DJ. Patients with transient ischaemic attack or minor stroke should be admitted to hospital. Stroke 2006;37:1137-8.

9. Lindley RI. Patients with transient ischaemic attack do not need to be admitted to hospital for urgent evaluation and treatment. Stroke 2006;37:1139-40.

10. The Intercollegiate Working Party for Stroke. National Clinical Guidelines for Stroke. London: RCPL, 2004.

11. Intercollegiate Stroke Working Party. National Sentinel Audit, 2006. http:// www.rcplondon.ac.uk/ (accessed 11 October 2007).

12. Hankey GJ, Warlow CP. Transient ischaemic attacks of the brain and eye. London: WB Saunders, 1994.

13. Hatano S. Experience from a multicentre stroke register: a preliminary report. Bull World Health Organ 1976;54:541-53.

14. Dennis MS, Bamford JM, Sandercock PAG, et al. A comparison of risk factors and prognosis for transient ischaemic attacks and minor ischaemic strokes. The Oxfordshire Community Stroke Project. Stroke 1989;20:1494-9.

15. van Swieten JC, Koudstaal PJ, Visser MC, et al. Interobserver agreement for the assessment of handicap in stroke patients. Stroke 1988;19:604-7.

16. Mahoney FI, Barthel DW. Functional evaluation: the Barthel index. Md State Med J 1965;14:61-5.

17. Prineas RJ, Crow RS, Blackburn H. The Minnesota Code Manual of Electrocardiagraphic Findings: standards and procedures for measurement and classification. Boston: John Wright, 1982.

18. Meschia JF, Brott TG, Chukwudelunzu FE, et al. Verifying the stroke-free phenotype by structured telephone interview. Stroke 2000;31:1076-80.

19. Fox KA. Acute coronary syndromes: presentation-clinical spectrum and management. Heart 2000;84:93-100.

20. Gladstone DJ, Kapral MK, Fang J, et al. Management and outcomes of transient ischaemic attacks in Ontario. Can Med Assoc J 2004;170:1099-104.

21. Lisabeth LD, Ireland JK, Risser JM, et al. Stroke risk after transient ischaemic attack in a population-based setting. Stroke 2004;35:1842-6.

22. Redgrave JNE, Coutts SB, Schulz UG, et al. Systematic review of associations between the presence of acute ischaemic lesions on diffusion-weighted imaging and clinical predictors of early stroke risk after transient ischaemic attack. Stroke 2007;38:1482-8.

23. Johnston SC, Sidney S, Bernstein AL, et al. A comparison of risk factors for recurrent TIA and stroke in patients diagnosed with TIA. Neurology 2003;60:280-5

24. Giles MF, Rothwell PM. Prediction and prevention of stroke after transient ischaemic attack in the short and long term. Expert Rev Neurother 2006;6:381-95.

25. Rothwell PM, Buchan AM, Johnston SC. Recent advances in management of transient ischaemic attacks and minor strokes. Lancet Neurol 2006;5:323-31. 


\section{APPENDIX}

Brain Injury Research Group, University of Manchester, UK PJ Tyrrell, NJ Rothwell, SJ Hopkins, A Vail, CJ Smith, JR Selvarajah, A Parry-Jones, RF Georgiou, S Hulme.

\section{Outcome Event Review and Adjudication Committee}

PJ Tyrrell, CS Sherrington, JR Staniland.

\section{Advisory Committee}

GA Ford, HS Markus.
NORTHSTAR Collaborating Centres and Investigators:

Salford Roval NHS Foundation Trust, UK

PJ Tyrrell, CS Sherrington, J Wainwright, EM Barberan.

Central Manchester University Hospitals NHS Trust, UK

G Subramanian.

Trafford Healthcare NHS Trust

S Musgrave.

Walton Centre for Neurology and Neurosurgery NHS Trust, UK

P Humphrey, TP Enevoldson, RP White, HCA Emsley.

Aintree University Hospitals NHS Foundation Trust, UK

AK Sharma.

\section{Neurological picture}

\section{Elongated styloid process (Eagle's syndrome) as a cause of atypical craniocervical pain}

A 48-year-old Caucasian woman presented with a long term history of oropharyngeal intermittent pain associated with foreign body sensation in her throat, and difficulty swallowing. The pain radiated to the right mastoid area and increased with neck rotation. Intraoral palpation triggered a painful sensation in the right tonsillar fossa. Three dimensional CT bone window mode volume-rendered reconstruction of the upper cervical region showed bilaterally elongated styloid processes, measuring $46 \mathrm{~mm}$ and $43 \mathrm{~mm}$ on the right and left, respectively (fig 1 ). The patient underwent a successful intra-oral styloidectomy and 3 years later she remains pain free.

Eagle's syndrome is characterised by an elongated styloid process or a calcified stylohyoid ligament which causes craniocervical and recurrent throat pain. ${ }^{1}$ Clinicians should consider this entity in the differential workup of a patient with atypical craniocervical pain. Three dimensional CT is a valuable diagnostic tool in Eagle's syndrome to make accurate length measurements of the styloid process. ${ }^{2}$

\section{J L Hernández, J Velasco}

Department of Internal Medicine, Hospital Marqués de Valdecilla, University of Cantabria Santander, Spain

Correspondence to: Dr J L Hernández, Department of Internal Medicine, Hospital Marqués de Valdecilla, University of Cantabria, 39008 Santander, Spain; mirhhj@humv.es

Competing interests: None.

J Neurol Neurosurg Psychiatry 2008;79:43. doi:10.1136/jnnp.2007.121020

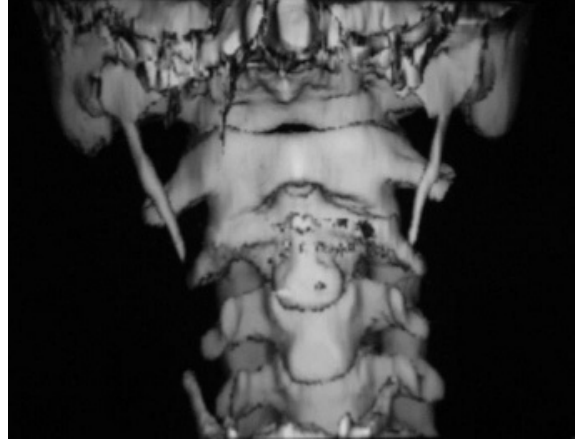

Figure 1 Three dimensional CT bone window mode volume-rendered reconstruction of the upper cervical region shows bilaterally elongated styloid process/consistent with Eagle's syndrome.

\section{REFERENCES}

1. Sun CK, Mercuri V, Cook MJ. Eagle syndrome: an unusual cause of head and neck pain. Arch Neurol 2006:63:294-5

2. Lee S, Hillel A. Three-dimensional computed tomography imaging of Eagle's syndrome. Am J Otolaryngol 2004;25:109. 